\title{
Valor preditivo da g'licemia de jejum pré-operatória de pacientes diabéticos quanto ao resultado cirúrgico da cirurgia de catarata
}

\author{
Predictive value of preoperative fasting glucose test of diabetic patients regarding \\ surgical outcome in cataract surgery
}

\author{
Maurício Abujamra Nascimento \\ Rodrigo Pessoa Cavalcanti Lira ${ }^{2}$ \\ Newton Kara-José ${ }^{3}$ \\ Carlos Eduardo Leite Arieta ${ }^{4}$
}

Trabalho realizado na Disciplina de Oftalmologia do Departamento de Oftalmo-Otorrinolaringologia Universidade Estadual de Campinas - UNICAMP.

${ }^{1}$ Pós-graduando, nível doutorado da Faculdade de Ciências Médicas da Universidade Estadual de Campinas UNICAMP. Campinas (SP).

${ }^{2}$ Professor Doutor colaborador da Pós-graduação da UNICAMP. Campinas (SP).

Professor Titular de Oftalmologia da UNICAMP e Faculdade de Medicina da Universidade de São Paulo - USP. São Paulo (SP).

${ }^{4}$ Professor Livre Docente em Oftalmologia pela UNICAMP. Campinas (SP).

Endereço para correspondência: Maurício Abujamra Nascimento - Rua Artur Freitas Leitão, 274 - Campinas (SP) CEP 13092-410

E-mail: mauabujamra@uol.com.br

Recebido para publicação em 20.01.2004

Versão revisada recebida em 06.10.2004

Aprovação em 13.12.2005

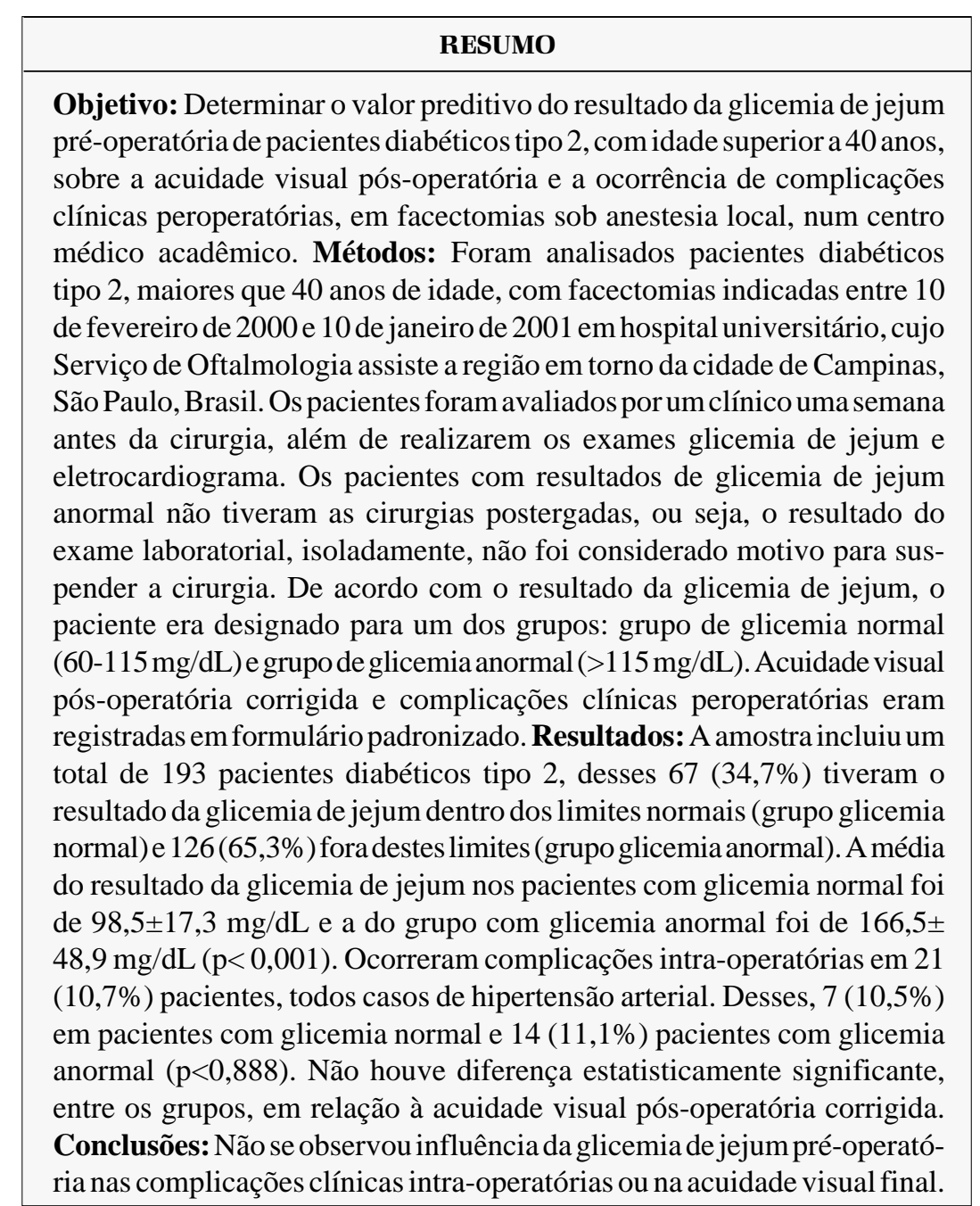

Descritores: Catarata; Glicemia; Jejum; Acuidade visual; Cuidados pré-operatórios; Valor preditivo; Idoso

\section{INTRODUÇÃO}

Indivíduos diabéticos desenvolvem catarata mais freqüentemente do que não diabéticos ${ }^{(1)}$. Estima-se que 16 milhões de pessoas nos EUA são diabéti$\cos ^{(2)}$. Apesar da principal indicação de facectomia em diabéticos ser a reabili- 
tação visual, muitos casos necessitam da cirurgia para complementação diagnóstica e tratamento adequado da retinopatia ${ }^{(3)}$.

Muitos estudos relacionam a cirurgia de catarata em diabéticos com resultados visuais pobres ${ }^{(4)}$. Alterações como fragilidade endotelial e iriana, aumento da permeabilidade vascular e maior inflamação, com deposição de fibrina, além de complicações pós-operatórias como sinéquias, edema macular cistóide, opacidade capsular e hemorragias intra-oculares, podem prejudicar a acuidade visual final e impedir o tratamento da retinopatia ${ }^{(5)}$. Alguns estudos sugerem o adiamento da cirurgia até o comprometimento severo da visão ${ }^{(6)}$. Em contrapartida, isso adia também a análise detalhada do fundo de olho e o tratamento, prejudicando o prognóstico visual a longo prazo ${ }^{(7)}$.

O diabetes de longa data está associado a alterações cardiovasculares, incluindo doença arterial coronariana, hipertensão, e mudanças na função autonômica, que contribuem para um aumento no risco cardiovascular do paciente diabéti$\operatorname{co}^{(8-9)}$. A associação entre diabetes e hipertensão está relacionada com elevação do risco de possíveis complicações peroperatórias $^{(10)}$, justamente devido ao aumento da chance da presença de lesões em órgãos-alvo como rim e coração.

O paciente cirúrgico tem alguns fatores hiperglicemiantes: a glicemia tende a elevar-se no período pré-operatório devido a uma combinação de estresse fisiológico e psíquico que elevam os níveis séricos de cortisol e epinefrina ${ }^{(11)}$, já no perioperatório há um aumento na taxa basal de metabolismo, hiperglicemia e aumento da necessidade de insulina. Nos diabéticos a deficiência de insulina piora o quadro de hiperglicemia, mas nas cirurgias eletivas não-complicadas este aumento é de apenas $10 \%$, sem repercussões sistêmicas importantes ${ }^{(8)}$.

A necessidade da realização deste estudo veio do fato de que nos deparamos por diversas vezes com um resultado de glicemia pré-operatória anormal, num diabético previamente controlado. Diante de tal quadro, somos por muitas vezes obrigados a adiar um procedimento que visa não só o restabelecimento visual do paciente, mas também a adequação dos meios ópticos para melhor diagnóstico e tratamento.

Existem várias formas de avaliar a função visual. Como parâmetro isolado, a mais utilizada é a medida de acuidade visual. Estudos envolvendo valores e variações de acuidade visual suscitam dificuldades operacionais e podem, dependendo da escala empregada nas quantificações, levar a interpretações discrepantes $^{(12-14)}$. Esta medida é um dado não-paramétrico, portanto deve ser avaliado na forma de classes, evitando-se o incorreto artifício de determinar médias ${ }^{(13)}$.

O presente estudo prospectivo teve por objetivo determinar o valor preditivo da glicemia de jejum pré-operatória de pacientes diabéticos tipo 2, com idade superior a 40 anos, sobre a acuidade visual pós-operatória e a ocorrência de complicações clínicas peroperatórias, em facectomias sob anestesia local, num centro médico acadêmico.

\section{MÉTODOS}

\section{Pacientes e procedimentos médicos}

O estudo foi desenvolvido em um hospital universitário, cujo Serviço de Oftalmologia assiste Campinas e região, SP, Brasil, realizando anualmente cerca de 2.000 facectomias eletivas. Foi aprovado pelo Comitê de Ética do hospital estudado.

Os pacientes diabéticos tipo 2 com indicação para cirurgia de catarata, foram recrutados entre 10 de fevereiro de 2000 e 10 de janeiro de 2001. A indicação cirúrgica existia a partir do momento em que a opacidade do cristalino tinha repercussões visuais importantes ou impedia exame e tratamento adequado retiniano. Todos os pacientes foram anestesiados com bloqueio peribulbar, utilizando-se $5 \mathrm{ml}$ de uma mistura 1:1 de bupivacaína $0,5 \%$ e lidocaína $2 \%$, com administração prévia de $5 \mathrm{mg}$ de diazepan via oral. Foram excluídos do estudo pacientes com menos de 40 anos de idade, pacientes diabéticos tipo 1, pacientes submetidos previamente à cirurgia ocular, pacientes com indicação de anestesia geral, ou pacientes que haviam sofrido infarto agudo do miocárdio até três meses antes da cirurgia. Todos os pacientes preencheram ficha de consentimento livre e esclarecido. Todos os pacientes foram submetidos à extração extracapsular realizada por 15 residentes de segundo ano em treinamento sob supervisão de médicos contratados da instituição.

Os pacientes foram avaliados por um clínico uma semana antes da cirurgia, além de realizarem os exames glicemia de jejum e eletrocardiograma. Foi adotada a classificação de condição clínica da American Society of Anesthesiology ${ }^{(15)}$.

Os pacientes com resultados de glicemia de jejum anormal não tiveram as cirurgias postergadas, ou seja, o resultado do exame laboratorial, isoladamente, não foi considerado motivo para suspender a cirurgia.

De acordo com o resultado da glicemia de jejum, o paciente era designado para um dos grupos: grupo de glicemia normal (60-115 mg/dL) ou grupo de glicemia anormal (>115 mg/dL).

\section{Coleta de dados}

Os dados pré-operatórios foram coletados por meio de uma ficha com dados da acuidade visual corrigida pré-operatória, história e exame físico, completado pelo médico clínico no momento da avaliação pré-operatória. Eventos médicos intra-operatórios foram registrados numa ficha de protocolo pelo oftalmologista responsável pela cirurgia ou por um membro da equipe de enfermagem, assim como a acuidade visual corrigida pós-operatória após três meses da cirurgia.

Os eventos médicos adversos (complicações clínicas préoperatórios) foram muito bem definidos ${ }^{(16)}$. Os eventos médicos foram revisados por dois especialistas (um clínico e um cardiologista) para determinar se eles estavam enquadrados nas definições pré-estabelecidas. Os revisores não tinham conhecimento do grupo a que pertencia o paciente. Eventos médicos que ocorreram no dia da cirurgia antes da alta do paciente foram considerados como relacionados à cirurgia. Não foram incluí- 
dos eventos clínicos ocorridos após a alta do paciente devido a dificuldades com o seguimento dos mesmos.

A acuidade visual corrigida pré e pós-operatória (AV) foi anotada de acordo com a classificação de cegueira da Organização Mundial de Saúde (WHO), consistindo de três classes: visão normal ou próxima da normal ( $\mathrm{AV} \geq 20 / 60)$, visão baixa $(20 / 400 \leq \mathrm{AV}<20 / 60)$ e cegueira $(\mathrm{AV}<20 / 400)^{(17)}$.

Foram citadas as patologias associadas com prevalência superior a $1 \%$ nos pacientes estudados.

Os parâmetros de normalidade para o teste de glicemia de jejum foram $60-115 \mathrm{mg} / \mathrm{dL}$. As anormalidades nos eletrocardiogramas foram definidas ${ }^{(18)}$.

\section{Tamanho da amostra e análise estatística}

Foi planejada uma amostra de 180 pacientes diabéticos tipo 2 (60 no grupo de pacientes diabéticos com glicemia normal e 120 no grupo de pacientes diabéticos com glicemia anormal). Assumido-se uma taxa de eventos médicos adversos de $10 \%$ no grupo de pacientes com glicemia normal, esta amostra permitiria $80 \%$ de poder para detectar uma diferença tão pequena quanto $19 \%$ no grupo de pacientes com glicemia anormal. Os resultados desta análise foram considerados significantes se o valor de $P$ fosse menor que 5\%(19-20).

A estimativa de facectomias em pacientes operados pela primeira vez, no período do estudo, era de 1.000 cirurgias, e destas $19 \%$ em indivíduos diabéticos tipo 2, gerando aproximadamente 190 candidatos ao estudo proposto.

Foram feitos testes para detectar diferenças entre variáveis, usando o teste do Qui-quadrado, ou o teste exato de Fisher quando indicado, para variáveis categóricas, além de análise de variância (one-way ANOVA) para variáveis contínuas. A análise estatística foi executada com o auxílio do software EpiInfo ${ }^{\mathrm{TM}} 2000$.

\section{RESULTADOS}

A amostra incluiu um total de 193 pacientes, desses 67 $(34,7 \%)$ tiveram o resultado da glicemia de jejum dentro dos limites normais (grupo glicemia normal) e $126(65,3 \%)$ fora destes limites (grupo glicemia anormal). A média do resultado da glicemia de jejum nos pacientes com glicemia normal foi de $98,5 \pm 17,3 \mathrm{mg} / \mathrm{dL}$ e a do grupo com glicemia anormal foi de $166,5 \pm 48,9 \mathrm{mg} / \mathrm{dL}(\mathrm{p}<0,001)$.

A média da idade nos pacientes com glicemia normal foi de $65 \pm 10$ anos e a do grupo com glicemia anormal foi de $66 \pm 11$ anos $(\mathrm{p}<0,428)$. Ambos os grupos também estiveram equilibrados quanto ao sexo, classificação clínica ASA, patologias sistêmicas associadas, resultado de eletrocardiograma e acuidade visual pré-operatória (Tabela 1).

Ocorreram complicações clínicas intra-operatórias em 21 $(10,7 \%)$ pacientes, todos casos de hipertensão arterial. Desses $7(10,5 \%)$ em pacientes com glicemia normal e $14(11,1 \%)$ pacientes com glicemia anormal $(\mathrm{p}<0,888)$. Não houve diferença estatisticamente significante, entre os grupos, em relação à acuidade visual pós-operatória corrigida (Tabela 2).

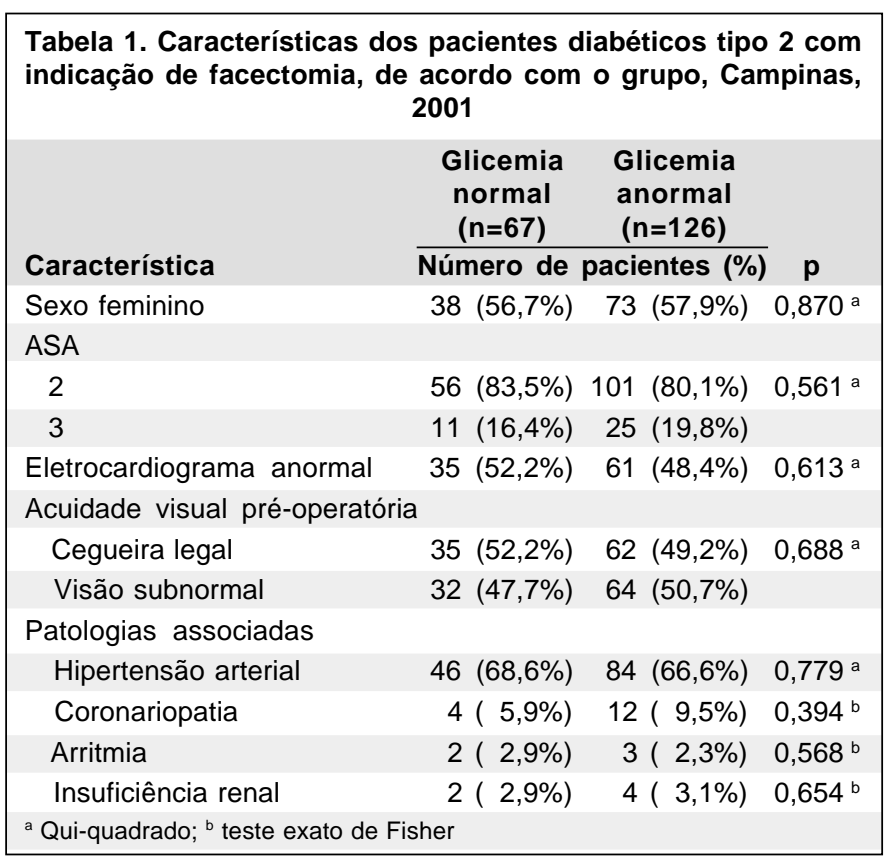

\begin{tabular}{|c|c|c|c|}
\hline \multirow[b]{2}{*}{ Característica } & $\begin{array}{c}\text { Glicemia } \\
\text { normal } \\
(n=67)\end{array}$ & $\begin{array}{c}\text { Glicemia } \\
\text { anormal } \\
(n=126)\end{array}$ & \multirow[b]{2}{*}{$\mathbf{P}$} \\
\hline & \multicolumn{2}{|c|}{ Número de pacientes (\%) } & \\
\hline Cegueira legal & $5(7,4 \%)$ & $8(6,3 \%)$ & $0,884^{a}$ \\
\hline Visão subnormal & $12(17,9 \%)$ & $20(15,8 \%)$ & \\
\hline Visão normal & $50(74,6 \%)$ & $98(77,7 \%)$ & \\
\hline a Qui-quadrado & & & \\
\hline
\end{tabular}

\section{DISCUSSÃO}

A cegueira por catarata atinge em torno de 25 milhões de pessoas no mundo ${ }^{(21-22)}$ e um crescimento deste número é esperado nas próximas décadas devido ao aumento da sobrevida da população(23-25). Estima-se que, no Brasil, $19 \%$ dos pacientes com catarata senil são diabéticos tipo $2^{(16)}$.

Diante de um quadro crônico de recursos limitados, não se deve poupar esforços para a equalização dos gastos com material de consumo cirúrgico e com a própria rotina operacional ${ }^{(26)}$. Com a percepção de que muitas cirurgias podiam ser realizadas sem internação do paciente com redução de custos e sem sacrificar a segurança, centros cirúrgicos ambulatoriais tem proliferado nos últimos $20 \operatorname{anos}^{(26-27)}$. Além disso, dogmas relativos à validade de testes médicos vêm sendo questionados, e o que era considerada uma ótima avaliação pré-operatória está sendo reavaliada a partir das reais necessidades médicas e relação custo-benefício ${ }^{(28-29)}$. A própria rotina de requisição indiscriminada de exames é questionável, havendo evi- 
dências de que a requisição seletiva dos testes seria uma conduta mais racional ${ }^{(16,29)}$.

A solicitação rotineira da glicemia de jejum pré-operatória é questionável, mesmo nos casos em que há uma justificativa baseada na literatura ${ }^{(30-31)}$. Tal interrogação se deve ao fato de que a quase totalidade das solicitações deriva da presença de diabetes, existindo mitos referentes à associação entre diabetes e cirurgia, e, na tentativa de evitar complicações cirúrgicas relacionadas a esta doença, como maior propensão à infecção e deiscência de suturas, valoriza-se de forma demasiada o controle da glicemia na época da cirurgia, quando na verdade o mais importante é o controle crônico ${ }^{(11,32-33)}$. Aliás, uma hiperglicemia moderada (até $250 \mathrm{mg} / \mathrm{dL}$ ) no perioperatório é preferível à possibilidade de ocorrer hipoglicemia ${ }^{(34-35)}$. Somese a isso o fato de que o estresse diante da perspectiva de cirurgia pode impedir que pacientes previamente controlados atinjam valores glicêmicos considerados ideais e já alcançados historicamente no seu controle rotineiro ${ }^{(11)}$.

Um estudo retrospectivo ${ }^{(34)}$, comparou morbidade pósoperatória entre diabéticos e não-diabéticos, em que os demais fatores como idade e co-morbidades foram pareados, tendo sido observado o mesmo número de complicações nestes dois grupos, não sustentando o mito de que diabetes por si só aumenta o risco cirúrgico ou que a hiperglicemia isoladamente seria um fator importante de morbidade pós-operatória em pacientes diabéticos. Este mesmo estudo também demonstrou que os níveis glicêmicos foram menores em pacientes diabéticos com complicações comparados a pacientes diabéticos sem complicações. Um outro estudo lembra que se deve evitar hiperglicemia acima de $250 \mathrm{mg} / \mathrm{dL}$ intra-operatória, entretanto o maior perigo é a hipoglicemia não reconhecida ${ }^{(11)}$.

Com os resultados apresentados neste estudo, percebe-se que as complicações clínicas peroperatórias observadas não mostraram relação com a glicemia pré-operatória, sugerindo que o resultado do exame isolado não é fator preditivo de eventos médicos adversos perioperatórios em facectomias com anestesia local.

Observamos também neste estudo que o resultado anormal da glicemia pré-operatória também não mostrou influência na acuidade visual final corrigida. Vários autores já vêm defendendo que a evolução ou não da retinopatia diabética após facectomia está mais relacionada com a evolução natural da doença, estágio prévio da retinopatia e controle glicêmico do que com o ato cirúrgico em si ${ }^{(36-37)}$.

\section{CONCLUSÃO}

Por este estudo ser pioneiro no assunto abordado, outros serão necessários para que sejam ratificadas as conclusões aqui observadas. Mas, de qualquer modo, estes resultados estimulam a solicitação seletiva da glicemia de jejum em diabéticos apenas quando fizer parte da rotina de acompanhamento crônico do paciente e não como um ato isolado de triagem pré- operatória. Isto será mais um passo para a diminuição dos custos deste procedimento, evitando cancelamentos desnecessários de cirurgias e sem diminuir a qualidade do serviço de saúde.

\section{ABSTRACT}

Objective: To determinate the predictive value of preoperative glucose test of diabetic patients, with age above 40 years, for visual acuity outcome and clinical perioperative complications, in cataract surgery with local anesthesia, in an academical medical center. Methods: Type 2 diabetic patients, above 40 years of age, indicated for cataract surgery between February 10, 2000 and January 10, 2001, at the State University of Campinas, São Paulo, Brazil. All patients had a preoperative medical assessment by a physician one week before surgery, and were submitted to serum fasting glucose test and 12-lead electrocardiogram. There was no delay in the surgeries of patients with abnormal serum glucose test results, so, the result of the test alone was not a reason to cancel the surgery. According to the glucose test, the patients were assigned to one of two groups: Normal Glucose Test Group $(60-115 \mathrm{mg} / \mathrm{dL})$ or Abnormal Glucose Test Group ( $>115 \mathrm{mg} / \mathrm{dL}$ ). Postoperative best-corrected visual acuity and clinical perioperative complications were recorded on a standardized form. Results: The sample consisted of 193 patients. 67 (34.7\%) had a serum glucose test within normal limits (normal glucose test group) and $126(65.3 \%)$ outside normal limits (abnormal glucose test group). The mean result of the "normal glucose test group" was $98.5 \pm 17.3 \mathrm{mg} / \mathrm{dL}$ and $166.5 \pm 48.9 \mathrm{mg} / \mathrm{dL}$ for the "abnormal glucose test group" $(\mathrm{p}<0.001)$. We observed perioperative complications in $21(10.7 \%)$ patients, all arterial hypertension cases; 7 (10.5\%) of these in patients with normal glucose test result and $14(11.1 \%)$ in patients with abnormal result $(\mathrm{p}<0.888)$. The postoperative best-corrected visual acuity was similar in both groups. Conclusion: There was no influence of the preoperative serum glucose level on perioperative clinical complications or visual acuity outcome.

Keywords: Cataracts; Blood glucose, fasting; Visual acuity; Preoperative care; Predictive value; Aged

\section{REFERÊNCIAS}

1. Klein BEK, Klein R, Moss SE. Incidence of cataract surgery in the Winscosin epidemiologic study of diabetic retinopathy. Am J Ophthalmol. 1995;119 (3):295-300.

2. Karam JH - Diabetes mellitus \& hypoglycemia. In: Tierney JR LM, McPhee SJ, Papadakis MA. Current medical diagnosis and treatment. 38ed. Stamford: Connecticut, Appleton \& Lange; 1999. p.1118-60.

3. Edwards MG, Schachat AP, Bressler SB, Bressler NM. Outcome of cataract operations performed to permit diagnosis, to determine eligibility for laser therapy, or to perform laser therapy of retinal disorders. Am J Ophthalmol. 1994;118(4):440-4

4. Benson WE, Brown GC, Tasman W, McNamara JA, Vander JF. Extracapsular cataract extraction with placement of a posterior chamber lens in patients with diabetic retinopathy. Ophthalmology. 1993;100(5):730-8.

5. Ionides A, Dowler JG, Hykin PG, Rosen PH, Hamilton AM. Posterior 
capsule opacification following diabetic extracapsular extraction. Eye. 1994;8(Pt 5):535-7.

6. Harding JJ, Egerton M, Van Heyningen R, Harding RS. Diabetes, glaucoma, sex and cataract: analysis of combined data from two case control studies. Br J Ophthalmol. 1993;77(1):2-6. Comment in: Br J Ophthalmol. 1993;77(7):464.

7. Dowler JG, Hykin PG, Lightman SL, Hamilton AM. Visual acuity following extracapsular cataract extraction in patients with diabetes: a meta-analysis. Eye. 1995;9(Pt 3):313-17.

8. Alberti KG, Thomas DJB. The management of diabetes during surgery. Br J Anaesth. 1979;51(7):693-709.

9. Haynes JM, Hodgson WC, Cooper ME. Rat amylin mediates a pressor response in the anaesthetised rat: implications for the association between hypertension and diabetes mellitus. Diabetologia. 1997;40(3):256-61.

10. Eagle KA, Brundage BH, Chaitman BR, Ewy GA, Fleisher LA, et al. Guidelines for preoperative cardiovascular evaluation for noncardiac surgery. Circulation. 1996;93(6):1278-317. Comment in: Circulation. 1997;95(2):530-1.

11. Siperstein MD - Special medical problems in surgical patients. In: Way LW Current surgical diagnosis and treatment. 10 ed., Connecticut: Appleton \& Lange; 1994. p.40-2.

12. Bicas HEA. Acuidade visual. Medidas e notações. Arq Bras Oftalmol. 2002; 65(3):375-84.

13. Wilson II FM. Practical ophthalmology. A manual for the beginning residents. San Francisco: American Academy of Ophthalmology; 1996. 432p.

14. Miller KM. Basic and clinical science course 2002. San Francisco: American Academy of Ophthalmology; 2002. 357p.

15. American Society of Anesthesiologists. New classification of physical status. Anesthesiology. 1963;24(2):111.

16. Lira RPC, Nascimento MA, Moreira-Filho DC, Kara-José N, Arieta CEL. Are routine preoperative medical tests needed with cataract surgery? Rev Panam Salud Publica. 2001;10(1):13-7.

17. World Health Organization. International classification of impairments, disabilities, and handicaps. Geneva: WHO; 1980.

18. Johnson H, Knee-Ioli S, Butler TA, Munoz E, Wise L. Are routine preoperative laboratory screening tests necessary to evaluate of ambulatory surgical patients? Surgery. 1988;104(4):639-45.

19. Daniel WW. Biostatistics: a foundation for analysis I the health sciences. 7ed., New York: Wiley; 1999. 755p.

20. Fleiss JL. Statistical methods for rates and proportions. 2ed., New York: Wiley; 1981. 352p.
21. Dawson CR, Schwab IR. Epidemiology of cataract - a major cause of preventable blindness. Bull Word Health Organ. 1981;59(4):493-501.

22. Schwab L. Cataract. In: Schwab L. Eye care in developing nations. 3.ed. San Francisco: The Foundation of the American Academy of Ophthalmology; 1999. p.21-52.

23. Apple DJ, Ram J, Foster A, Peng Q. Blindness in the world. Surv Ophthalmol. 2002;45(Suppl 1):S21-31.

24. Blay SL. Envelhecimento populacional: panorama demográfico. J Bras Psiquiatr. 1991;40(7):361-4.

25. Wilson J. Clearing the cataract backlog. Br J Ophthalmol. 1987;71(2):158-60.

26. Kara-José N, Delgado AMN, Arieta CEL. Exeqüibilidade da cirurgia de catarata em hospital-escola: em busca de um modelo econômico. Rev Assoc Med Bras. 1994;40(3):186-8.

27. Arieta CEL, Kara-José N, Carvalho-Filho DM, Alves MR. Optimisation of a cataract-patient care service in Campinas, Brazil. Ophthalmic Epidemiol. 1999;6(1):1-11.

28. Roizen MF. More preoperative assessment by physicians and less by laboratory tests. N Engl J Med. 2000;342(3):204-5. Comment on: N Engl J Med. 2000;342(3): 168-75.

29. Schein OD, Katz J, Bass EB, Tielsch JM, Lubomski LH, Feldman MA, et al. The value of routine preoperative medical testing before cataract surgery. $\mathrm{N}$ Engl J Med. 2000;342(3):168-75. Comment in: ACP J Club. 2000;133(2):60.

30. MacPherson DS. Preoperative laboratory testing: should any tests be "routine" before surgery? Med Clin North Am. 1993;77(2):289-308.

31. Meyer P, Roizen MF, Murray W, Gronert G, Grogono A, Duke P. Who needs an ECG or a blood glucose test preoperatively: use of technology to improve selection [abstract]. Anesthesiology. 1991;75(Suppl 3A):A441.

32. Eckersley JR, Dudley HA. Wounds and wound healing. Br Med Bull. 1988; 44(2):423-36.

33. Hjortrup A, Rasmussen BF, Kehlet H. Morbidity in diabetic and non-diabetic patients after major vascular surgery. Br Med J. 1983;287(6399):1107-9.

34. Hjortrup A, Sorensen C, Dyremose E, Hjortso NC, Kehlet H. Influence of diabetes mellitus on operative risk. Br J Surg. 1985;72(10):783-5.

35. Tambascia M. Diabete e cirurgia. Rev Soc Cardiol Estado de São Paulo 2000;10:289-92.

36. Henricsson M, Heijil A, Janzon L. Diabetic retinopathy before and after cataract surgery. Br J Ophthalmol. 1996;80(9):789-93. Comment in: Br J Ophthalmol. 1996;80(9):778-9.

37. Dowler JG, Sehmi KS, Hykin PG, Hamilton AM. The natural history of macular edema after cataract surgery in diabetes. Ophthalmology. 1999;106 (4):663-8.

\section{CONGRESSO DA SOCIEDADE BRASILEIRA DE VISÃO SUBNORMAL}

\section{4 a 26 de Junho de 2005 \\ Unique Hotel - São Paulo - SP}

IN FO RMAÇÕES: Tel.: (11) 3266-4000 c/ Fabrício

E-mail: congresso@ visaosubnormal.org.br

Home-page: www.visa osubnormal.org.br 